\title{
Conduction velocity and refractory period of single motor nerve fibres in antecedent poliomyelitis
}

\author{
KRISTIAN BORG, JÖRGEN BORG \\ From the Department of Neurology, Karolinska Hospital, Stockholm, Sweden
}

SUMMARY The axonal conduction velocity and the axonal refractory period were determined using electromyographic techniques for 60 single motor units in 11 patients with antecedent poliomyelitis. The results were compared with previous observations in healthy subjects as well as subjects with motor neuron disease. In antecedent poliomyelitis there was a reduced proportion of nerve fibres with low conduction velocity as compared with the findings in healthy subjects. The mean value of the axonal conduction velocities in antecedent poliomyelitis $(42.4 \pm 3.7 \mathrm{~m} / \mathrm{s}$, $\mathrm{M} \pm \mathrm{SD}$ ) was significantly higher $(\mathrm{p}<0.01)$ while in motor neuron disease the corresponding value $(35.2 \pm 7.8 \mathrm{~m} / \mathrm{s})$ was significantly lower $(\mathrm{p}<0.005)$ than in healthy subjects $(39.8 \pm 4.9 \mathrm{~m} / \mathrm{s})$. In antecedent poliomyelitis the conduction velocity and refractory period were related as in healthy subjects while abnormally long refractory periods were observed in motor neuron disease.

In patients with motor neuron loss due to antecedent poliomyelitis the maximal motor nerve conduction velocity is normal or only slightly reduced. ${ }^{1}$ Remaining motor units have increased muscle territory ${ }^{23}$ and impaired neuromuscular transmission. ${ }^{45}$ Some patients suffer from late deterioration and a late onset, progressive muscular atrophy has been described. ${ }^{6}$ An increased frequency of motor neuron disease has been suggested ${ }^{78}$ but conclusive data concerning a causal relationship between prior poliomyelitis and late motor neuron disease are lacking. ${ }^{9}$

Previously the axonal conduction velocity and refractory period of single human motor units have been studied using electromyographic techniques. Data from young and elderly healthy subjects have been reported ${ }^{10-13}$ and abnormal axonal conduction velocity and refractory period have been described in motor neuron disease. ${ }^{14}$ The present study of patients with antecedent poliomyelitis was performed for comparison with these previous results taking also into regard the suggested association between motor neuron disease and antecedent poliomyelitis.

\section{Materials and methods}

The study included 11 patients aged 34-69 years with acute

Address for reprint requests: Dr Kristian Borg, Department of Neurology, Karolinska Hospital, S-104 01 Stockholm, Sweden.

Received 7 February 1986 and in revised form 24 June 1986. Accepted 25 June 1986 poliomyelitis at 3-22 years of age. The latency between the acute illness and the investigation was 28-51 years. Six patients described recent functional deterioration but none exhibited signs of motor neuron disease. All exhibited paresis of foot extensor muscles on one or both sides ranging from slight to subtotal.

Electromyographic recordings were made from the short toe extensor muscle by conventional bipolar needle electrodes (DISA Electronic, Skovlunde, Denmark). Selective motor unit recordings were possible owing to loss of motor units and increased muscle fibre density of remaining motor units, permitting the test motor unit potential to be identified at maximal voluntary contraction and after supramaximal nerve stimulation. The motor unit potentials were amplified and displayed on a Medelec oscilloscope no 4329 and recorded on Kodak Linagraph Direct Print Paper. The peroneal nerve was stimulated proximally, at the fibular head, and distally, at the ankle. Stimuli were delivered through surface electrodes $0.6 \mathrm{~cm}$ in diameter. The cathode was placed over the nerve trunk and the anode $2-3 \mathrm{~cm}$ laterally or medially to the cathode. Rectangular pulse waves of $0.2 \mathrm{~ms}$ duration were used. Stimulus strength could be gradually changed from $0-100 \mathrm{~mA}$. The test motor unit potential was identified by its size and shape and all-or-none appearance. Electrical stimuli were delivered as follows: (1) A single nerve stimulus was delivered proximally and distally. The latency difference for the test motor unit responses was calculated and the axonal conduction velocity was determined, (2) A single proximal and a single distal stimulus were then delivered coupled to each other. Stimulus strength was $10 \%$ above the axonal threshold at rest. The proximal stimulus was initially delayed $10-15 \mathrm{~ms}$ to permit the distally evoked, antidromic propagated nerve impulse to pass the proximal test point before stimulation. The stimulus 
interval was then reduced by $0.2 \mathrm{~ms}$ intervals until the second evoked motor unit potential disappeared. This was due to blocking at the proximal testing point during the refractory period after the antidromic impulse. By comparing the shortest interval without blocking with the previously determined latency difference (point 1 above) the axonal refractory period following an antidromic propagated nerve impulse was calculated. ${ }^{12}$

Room temperature was $23^{\circ}-25^{\circ}$. Skin temperature was continuously controlled and held at $32^{\circ} \mathrm{C}$ by DISA heater (DISA Electronic, Skovlunde, Denmark).

\section{Results}

Figure 1 shows the recordings of a test motor unit potential. In A the potential is recorded after distal nerve stimulation and in B after proximal nerve stimulation. The latency difference was $8.3 \mathrm{~ms}$, which for this motor unit corresponded to a conduction velocity
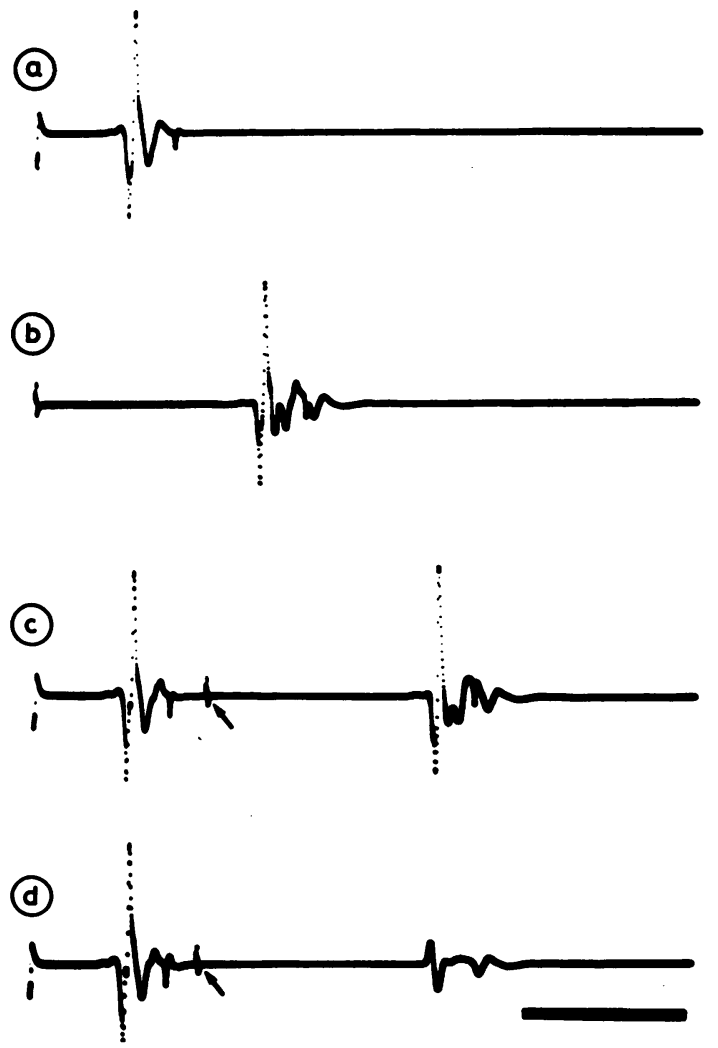

Fig 1 Electromyographic single motor unit recordings after delivering (a) one distal nerve stimulus, (b) one proximal nerve stimulus, (c) one distal and one proximal nerve stimulus coupled to each other with the proximal stimulus delayed $10 \cdot 2 \mathrm{~ms}$ and $(\mathrm{d})$ with the proximal stimulus delayed $10.0 \mathrm{~ms}$. Arrows indicate stimulus artefact of the proximal, delayed stimulus. Time bar $10 \mathrm{~ms}$. of $41 \mathrm{~m} / \mathrm{s}$. In C-D the blocking technique used to measure the axonal refractory period was applied (cf Methods). In C, the distal stimulus was delivered $10.2 \mathrm{~ms}$ prior to the proximal stimulus and no blocking occurred. In $\mathrm{D}$ the distal stimulus was delivered $10.0 \mathrm{~ms}$ prior to the proximal stimulus. At this stimulus interval the nerve fibre was still refractory after the antidromic impulse when the proximal stimulus was delivered and no second motor unit response occurred. Thus, for this nerve fibre the axonal refractory period at the proximal test stimulus point was $1.9 \mathrm{~ms}(10 \cdot 2-8 \cdot 3)$. The axonal conduction velocity and refractory period were determined for 60 motor units in 11 patients, 3-12 motor units being studied in each patient.

\section{Axonal conduction velocity}

Figure 2 shows the axonal conduction velocities of the 60 motor units together with corresponding data from 60 motor units in motor neuron disease, ${ }^{14} 120$ motor units in young healthy subjects (aged 25-45 years) ${ }^{10}$ and 102 motor units in elderly healthy subjects (aged 65-80 years). ${ }^{11}$ The axonal conduction
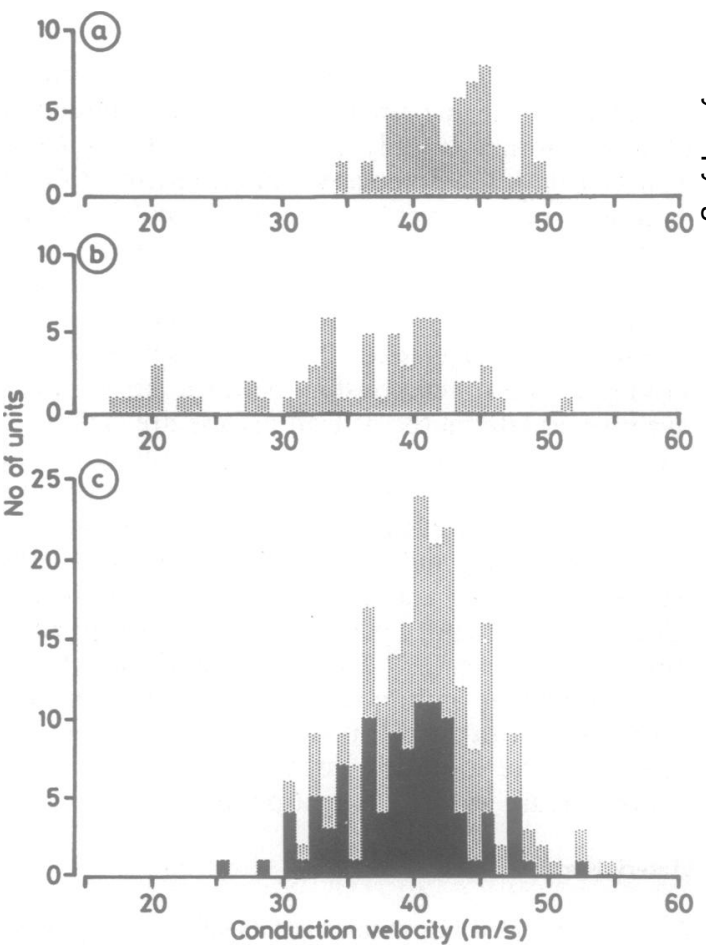

Fig 2 Axonal conduction velocities of (a) 60 motor units in antecedent poliomyelitis, (b) 60 motor units in motor neuron disease and (c) 222 motor units in healthy subjects. Black area indicates the results from elderly healthy subjects. Further description in text. 
velocity range was $34-49 \mathrm{~m} / \mathrm{s}$ for the polio patients, $25-54 \mathrm{~m} / \mathrm{s}$ for the healthy subjects and $17-51 \mathrm{~m} / \mathrm{s}$ in motor neuron disease. In the polio patients $3 \%(2 / 60)$ of the motor units had axonal conduction velocity below $35 \mathrm{~m} / \mathrm{s}$ while the corresponding figure in healthy subjects was $15 \%(34 / 222)$ and in motor neuron disease $40 \%(24 / 60)$. The mean value of the axonal conduction velocities in the polio patients was $42.4 \pm 3.7 \mathrm{~m} / \mathrm{s}(M \pm S D)$ which was significantly higher $(\mathrm{p}<0.01)$ than corresponding value in healthy subjects $(39.8 \pm 4.9)$ while the mean value of the axonal conduction velocities in motor neuron disease $(35.2 \pm 7.8)$ was significantly lower $(p<0.005)$ than in healthy subjects.

\section{Axonal refractory period}

The axonal refractory periods of the 60 motor units ranged $1.4-2.9 \mathrm{~ms}$. For less than $2 \%(1 / 60)$ of the motor units the axonal refractory period was longer than $2.5 \mathrm{~ms}$. In healthy subjects this was observed for less than $1 \%(1 / 109)$ of the motor units while in motor neuron disease $38 \%(23 / 60)$ of the motor units had axonal refractory periods longer than $2.5 \mathrm{~ms}^{14}$

Figure 3 shows the individual mean values of the axonal refractory periods and the axonal conduction velocities for each of the 11 polio patients together with corresponding data for 60 motor units from 17 patients with motor neuron disease ${ }^{14}$ and for 109 motor units from 22 healthy subjects. ${ }^{13}$ For the polio patients these refractory period data exhibited a mean of $1.8 \pm 0.24 \mathrm{~ms}$. Considering the inverse relationship between the axonal conduction velocity and axonal refractory period observed in healthy subjects, ${ }^{13}$ and that the axonal conduction velocities were higher in the polio patients, this mean value was not significantly different $(p>0.05)$ from corresponding value in healthy subjects $(1.5 \pm 0.26)$. In motor neuron disease the refractory period mean value $(2.3 \pm 0.46)$ was significantly higher $(p<0.01)$ than corresponding value in healthy subjects.

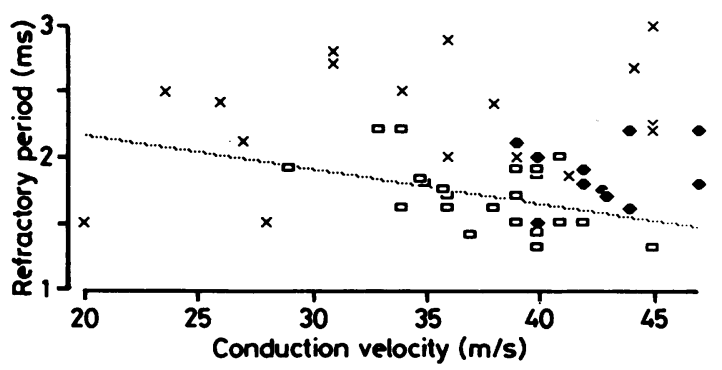

Fig 3 Axonal conduction velocity and refractory period. Mean values for 11 patients with antecedent poliomyelitis (dots), 17 patients with motor neuron disease (crosses) and 22 healthy subjects (boxes). Regression line for data for healthy subjects is inserted.

\section{Discussion}

For patients with antecedent poliomyelitis the conduction velocity and the refractory period of single motor nerve fibres were related as for healthy subjects, in contrast to the observations in motor neuron disease. In a previous study of patients with motor neuron disease $50 \%$ of the motor units had lower axonal conduction velocity and/or a longer axonal refractory period than that observed in healthy subjects. ${ }^{14}$ The present study showed no similar abnormalities in patients with antecedent poliomyelitis. It must be emphasised that none of the patients in this study had clinical signs of motor neuron disease. No patient with antecedent poliomyelitis and motor neuron disease was available for investigation. However, only where the abnormalities observed in motor neuron disease were found in a patient with prior poliomyelitis but without signs of motor neuron disease, would this be an argument for an association between antecedent poliomyelitis and motor neuron disease.

In patients with antecedent poliomyelitis there was a reduced proportion of motor units with low axonal conduction velocity as compared with patients with motor neuron disease and with healthy subjects. In healthy subjects low threshold motor units have low axonal conduction velocity. ${ }^{10}$ It might be argued that loss of motor units due to previous poliomyelitis makes it easier to identify and make recordings from high threshold motor units explaining why motor units with low axonal conduction velocity were relatively less common in antecedent poliomyelitis. However, in motor neuron disease there is a corresponding loss of motor units. In the motor neuron disease study, reduced recruitment of high threshold motor units due to upper motor neuron involvement was considered to be of minor importance as judged from motor unit firing rates and the results when supramaximal nerve stimuli were used. Further, in previous studies of healthy subjects special arrangements were made to obtain recordings from high threshold motor units. ${ }^{10}$ Thus, the reduced proportion of motor units with low axonal conduction velocity in antecedent poliomyelitis cannot be explained in terms of a methodological bias.

A reduced proportion of motor units with low axonal conduction velocity in antecedent poliomyelitis might be due to selective involvement of small motor neurons by the primary disease process or to secondary, adaptative mechanisms. It is an old clinical observation that motor activity increases the risk of developing paresis due to acute poliomyelitis but there are no data available confirming that more active low threshold motor units are affected by the acute disease to a greater extent.

Owing to axonal sprouting, remaining motor units 
have increased muscle territory in antecedent poliomyelitis. $^{23}$ It is not known whether this leads to increased functional demands influencing the motor neuron properties studied here. It must also be considered whether there is increased utilisation of remaining motor units and if this leads to changed motor neuron properties. Animal experiments have shown effects of increased motor activity on the dimensions of the motor neuron soma. ${ }^{15}$ In man, overuse of remaining motor units have been shown during locomotion in peroneal palsy. ${ }^{16}$ If this occurs during the long lasting state after prior poliomyelitis, it might have consequences for the motor neuron properties (work in progress).

In summary this study of patients with antecedent poliomyelitis has shown (1) axonal conduction velocities and axonal refractory periods of single motor units within the same range as in healthy subjects in contrast to previous findings in motor neuron disease and (2) a reduced proportion of motor units with low axonal conduction velocity as compared with the proportion in healthy subjects.

This study was supported by grants from the Swedish Medical Research Council (nr 84-14X-04749-09A), the Vivian Smith Foundation for Restorative Neurology and the Swedish Society for Traffic and Poliomyelitis Disabled.

\section{References}

1 Henriksen JD. Conduction velocities of motor nerves in normal subjects and patients with neuromuscular disorders. M.S. (Physical Medicine) Thesis: University of Minnesota. 1956.

2 Wohlfart G. Collateral regeneration in partially denervated muscles. Neurology 1958;8:175-80.
3 Coers C, Woolf AL. The Innervation of Muscle, a Biopsy Study. Oxford: Blackwell, 1959:65.

4 Hodes R. Electromyographic study of neuromuscular transmission in human poliomyelitis. Arch Neurol Psychiat 1948;60:457.

5 Stålberg E, Trontelj J. Single Fibre Electromyography. Old Woking, Surrey, UK: Mirvalle Press, 1979:118.

6 Campbell AM, Williams ER, Pearce J. Late motor degeneration following poliomyelitis. Neurology 1969;19:1101-6.

7 Zilkha KJ. Contribution to discussion on motor neurone disease. Proc $R$ Soc Med 1962;55:1028.

8 Poskanzer DC, Cantor HM, Kaplan GS. The frequency of preceding poliomyelitis in amyotrophic lateral sclerosis. In: Norris FH, Kurland LH, eds. Motor Neuron Diseases. New York: Grune and Stratton, 1969:286-90.

9 Alter M, Kurland LT, Molgaard CA. Late progressive Muscular Atrophy and Antecedent poliomyelitis. In: Advances in Neurology vol. 36, Rowland LP, ed. New York: Raven Press, 1982:303-9.

10 Borg J, Grimby L, Hannerz J. Axonal conduction velocity and voluntary discharge properties of individual short toe extensor motor units in man. $J$ Physiol (Lond) 1978;277:143-52.

11 Borg J. Properties of single motor units of the extensor digitorum brevis in elderly humans. Muscle Nerve 1981;4:429-32.

12 Borg J. Axonal refractory period of single short toe extensor motor units in man. $J$ Neurol Neurosurg Psychiatry 1980;43:917-24.

13 Borg J. Refractory period of single motor nerve fibres in man. J Neurol Neurosurg Psychiatry 1984;47:344-8.

14 Borg J. Conduction velocity and refractory period of single motor nerve fibres in motor neuron disease. $J$ Neurol Neurosurg Psychiatry 1984;47:349-53.

15 Edström JE. Effects of increased motor activity on the dimensions and the staining properties of the neuron soma. J Comp Neurology 1957;107:295-303.

16 Grimby L, Holm K, Sjöström L. Abnormal use of remaining motor units during locomotion in peroneal palsy. Muscle Nerve 1984;7:327-31. 